\title{
2006-755: AN ALTERNATIVE PARADIGM FOR ENGINEERING HOMEWORK: THE CASE OF ENGINEERING ECONOMICS
}

\section{John Ristroph, University of Louisiana-Lafayette}

JOHN H. RISTROPH is jointly appointed as a professor of Engineering and Technology

Management and as a professor of Economics and Finance at the University of Louisiana at Lafayette. His doctorate is in industrial engineering and operations research, and his non-academic experience includes service to the Louisiana Department of Natural Resources first as Head of Economics and Statistics and then as Director of Policy and Planning. 


\title{
An Alternative Paradigm for Engineering Homework: The Case of Engineering Economics
}

\begin{abstract}
Homework is an absolutely crucial component of engineering education, but there has been little progress in this vital area other than improvements in computational aids. This paper offers a new and thought-provoking paradigm for engineering homework by establishing the feasibility of routinely assigning problems with the same structure but unique numbers for each student. It examines the current status of homework, reports on a survey of students' needs, examines basic issues that affect widespread implementation, and considers meaningful enhancements for professors, such as automated grading and help for students.
\end{abstract}

\section{Introduction}

A common ground shared by engineering professors and their students is that neither are enamored of homework. Professors are keenly aware of its benefits, but some rediscover prayer while seeking assistance in grading. Students know that homework is necessary, but sometimes that necessity has more to do with getting a grade rather than a burning desire for long-term learning. This leads to a variety of compliance and evasion strategies that are all too familiar to professors. This paper first examines the current status of homework within engineering education. Then it presents a survey of what students think of alternatives to this status quo. This is followed by a closer look at the most promising alternative paradigm, how it can be implemented, and enhancements that benefit both professors and students.

\section{Current Status}

At a very general level, the traditional mode of engineering education consists of enlightened lectures, reinforcement and extension of the lectures via homework and labs, assessment of how much students learned, and evaluation of how well professors did their jobs. The homework step is critical, but onerous to all parties. Professors seek to reduce time spent on grading homework, and many students also develop methods to converse their time. Perhaps the most common student strategy is some form of copying. Working together does encourage cooperation and possibly increases communication skills, but weaker students frequently do not develop the independence that they will need at a professional level, or even to succeed on the next test.

How can simple copying be eliminated, while still allowing the best aspects of cooperation? One approach used by some professors is to give short, weekly tests. The benefit of this strategy is that it strongly discourages accumulating a learning deficit by not doing homework, and it provides rapid feedback that discourages copying. The cost is the class time that it consumes, as well as the time required for preparing and grading tests, and many professors feel that the costs outweigh the benefits. 
A more moderate strategy is to pick up and grade randomly selected homework problems and check the rest for completion. Problems of copying and inappropriate collaboration still exist, but nonetheless this approach's monitoring and feedback generally are helpful. A difficulty is that feedback is limited to only the selected problem(s) and to the degree that the professor expends time writing comments on the homework.

Students still seek help with homework during professors' office hours, but that practice has tended to evolve to the posting of solutions. Factors affecting both professors and students have contributed to this trend. Professors spend more time conducting research and raising funds, and students' jobs can make it difficult to use office hours. Postings can occur before or after due dates using either paper or the Internet. In any case, and it does not take long before homework files are created and exchanged. It is appropriate to explore alternatives to current homework practices, and the next section presents a feasibility study consisting of a survey that solicited students' input.

\section{Survey}

Students in two engineering economics sections learned of their progress in the course by receiving the results of their first test shortly before receiving the email survey shown in Table 1. Each of the test's ten problems was based on a homework question. The results were clearly bimodal, with $54 \%$ of the students earning A's or B's and $41 \%$ having D's or F's. The design of the test should have rewarded mastery of the homework problems, but Figure 1 shows only a 21\% correlation between homework and test scores, a value not significantly different from 0.0 (with a $\mathrm{P}$ value of 0.24.) Students had access to answers and solutions to homework before its due date both in the form of homework files maintained by students and via solutions posted by the professor, so it is possible that the lack of correlation is due to an unwise use of those resources. This a situation all too familiar to professors.

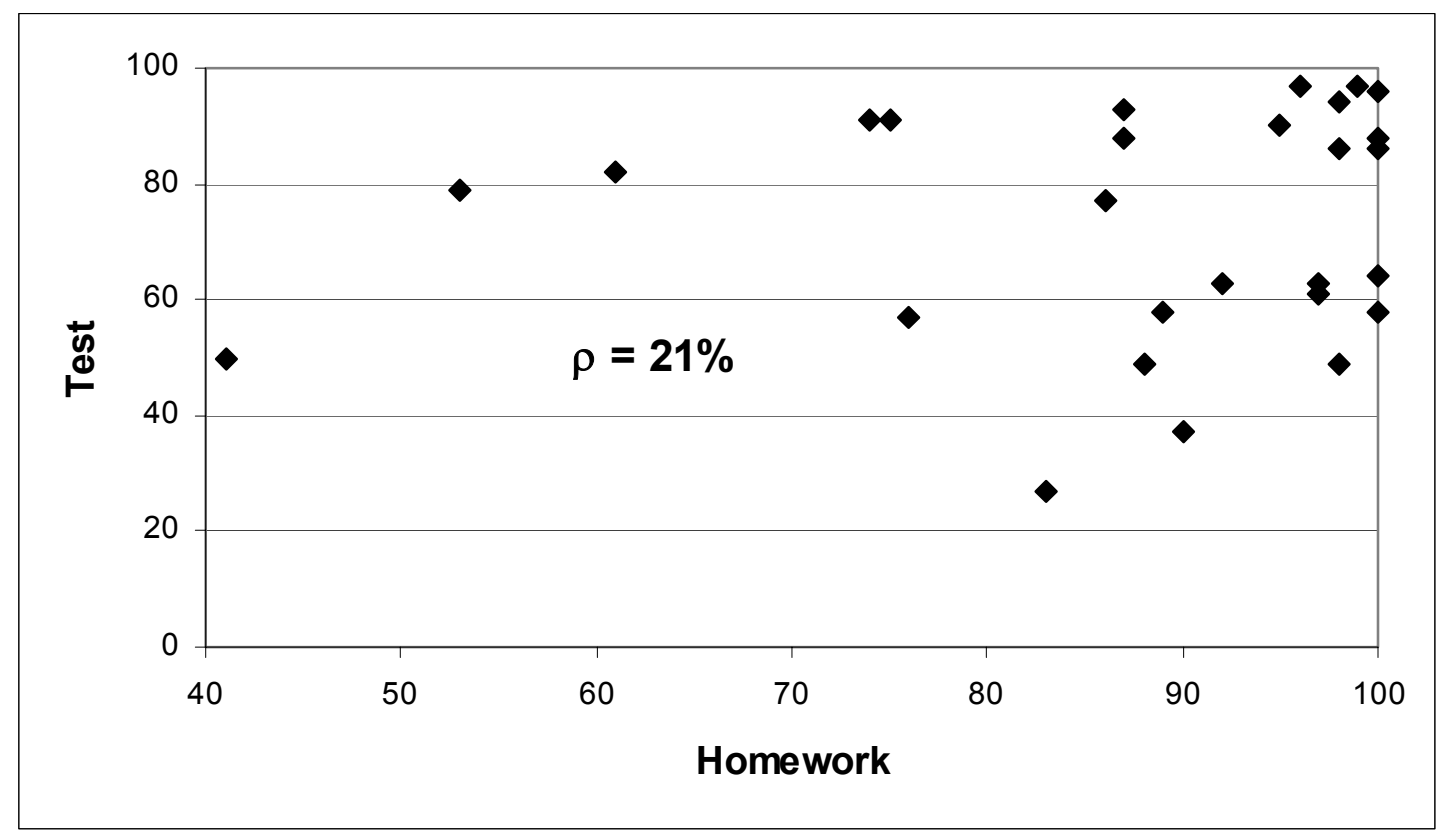

Figure 1. Homework and Test Scores 


\section{Table 1. Survey}

Please use the following scale in providing the numeric responses requested below. You are encouraged to also write any comments that you want.

\section{Strongly agree 2. Agree 3. Indifferent 4. Disagree 5. Strongly disagree}

1. Some engineering colleges require students to own laptops. Do you think that this would benefit students at ULL?

2. Recent software developments make it possible for each student to have his or her own homework problems. Each student would use the Web to download an Excel workbook that essentially has the same questions, but with different numbers. Please read the following alternatives carefully and indicate whether you think that this is a good idea IF:

a. No answers or solutions are available.

b. Answers are provided for some of each student's problems, but no solutions.

c. Answers are provided for some of all of each student's problems, but no solutions.

d. Answers are provided for some of each student's problems, and solutions are provided for some of each student's problems(before the homework's due date).

e. Answers are provided for all of each student's problems, and olutions are provided for each problem, but the solution uses ifferent numbers than the student's problem.

f. Answers are provided for all of each student's problems; solutions are provided for each problem (with different numbers than the student's problem); and after the homework is turned in, then solutions for each student's problems are provided (using the numbers on each student's problems).

g. Answers are provided for all of each student's problems, and solutions are provided for each problem (using the numbers on each student's problems).

h. Individualized problems are not a good idea under any circumstances.

i. Which of the above alternatives do you like the most?

3. Another recent software innovation also allows providing similar questions with unique numbers for each student, plus possibly having prompts to guide the student through the solution. This software allows a student to answer the question without prompts, and immediately move on to the next question if the answer is correct. Otherwise prompts will guide the student through the current problem, and then the numbers will be changed so the student can repeat the problem until he or she gets it right. This software never provides answers or solutions; it just indicates whether responses are correct or incorrect and gives feedback on incorrect answers.

a. Do you prefer the best alternative from survey question 2 above or the software in this survey question (number 3)?

b. Why? 
Table 2. Average Responses and Frequencies of Survey Responses

\begin{tabular}{|c|c|c|c|c|c|c|c|c|c|c|}
\hline & & Ques & ion & & $\stackrel{+\infty}{Z}$ & 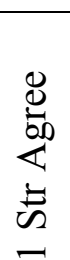 & $\underset{d}{\stackrel{\Xi}{\Delta}}$ & 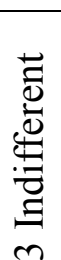 & 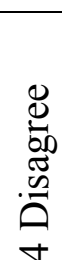 & 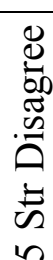 \\
\hline & Owning & laptops $b$ & eneficial & & 2.4 & 8 & 12 & 4 & 6 & 1 \\
\hline & Individu & alized so & utions: & & & & & & & \\
\hline & \begin{tabular}{|c|} 
Ans \\
Before
\end{tabular} & $\begin{array}{l}\text { Sol'n } \\
\text { Before }\end{array}$ & $\begin{array}{c}\text { Type } \\
\text { Before }\end{array}$ & $\begin{array}{l}\text { Sol'n } \\
\text { After }\end{array}$ & & & & & & \\
\hline $\mathrm{a}$ & No & No & & & 4.4 & 0 & 1 & 2 & 11 & 17 \\
\hline $\mathrm{b}$ & Some & No & & & 3.7 & 2 & 4 & 5 & 11 & 9 \\
\hline $\mathrm{c}$ & All & No & & & 3.0 & 5 & 8 & 6 & 6 & 6 \\
\hline $\mathrm{d}$ & Some & Some & Unique & & 3.1 & 2 & 8 & 10 & 6 & 5 \\
\hline $\mathrm{e}$ & All & All & Generic & & 2.2 & 9 & 13 & 4 & 4 & 1 \\
\hline $\mathrm{f}$ & All & All & Generic & Unique & 1.8 & 16 & 8 & 4 & 2 & 1 \\
\hline $\mathrm{g}$ & All & All & Unique & & 2.9 & 7 & 6 & 7 & 5 & 6 \\
\hline h & Individ & alized n & ver good & & 3.3 & 4 & 5 & 9 & 4 & 9 \\
\hline
\end{tabular}

Table 2 shows the responses of thirty-one students who responded to the survey that used a five point scale with 1 indicating strong agreement and 5 representing strong disagreement. Chisquared tests of independence and correlation analyses provided no indication that performance on the test affected responses to any question. This suggests that responses were fairly objective, or minimally that they were not emotional reactions to the test results.

The first question asked if the college should require students to own laptops, and it was designed to gauge students' outlook toward computer usage. Students generally favored the use of laptops, with $65 \%$ responding 1 or 2 to the statement that mandatory laptops would be beneficial. The $23 \%$ who responded unfavorably ( 4 or 5 ) frequently cited concern about the cost.

The second question noted that recent software developments make it possible for each student to have his or her own homework problems. Student can use the Web to download an Excel workbook that essentially has the same questions, but with different numbers. Given this capability, students were presented with alternative methods $a$ through $h$ (defined in the questionnaire) of computer-based assistance ranging from no aid to very extensive help. The average responses indicate a level of approval for each method, and they are plotted in Figure 2. They reveal that students want some help, but they recognize that too much assistance is not desirable.

Question 2.i confirmed the trend shown in Figure 2 by asking each student which method he or she liked the most. The relative frequencies with which each method was chosen are shown in Table 3, where there is a clear preference method $f$. This alternative provides homework problems to each student that have the same structure but unique numbers. Answers to each question are given before the due date, plus the complete solution of a problem with different numbers. After the due date, complete solutions are provided for each student's individual problems. 


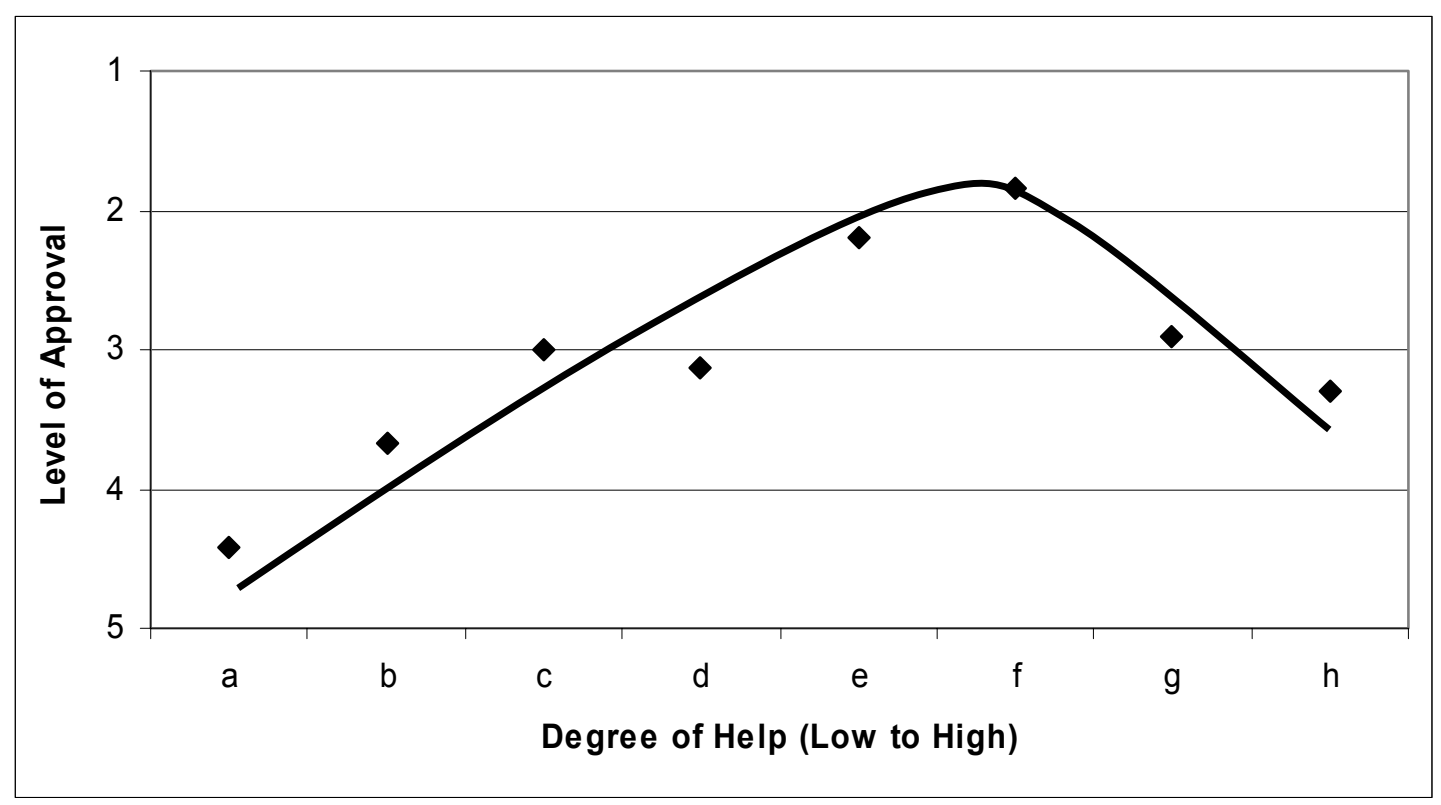

Figure 2. Average Responses to Question 2.

Question 3 asked each student to choose between another new software system and his or her preferred method from question 2 . The new alternative also provides individualized problems having common structures but unique numbers for each student. If the student enters a correct answer, then the software simply moves to the next problem. Otherwise prompts will guide the student through the current, incorrectly answered problem. Then the numbers are changed, and the student repeats the problem until he or she gets it right. This system never provides answers or solutions; it just indicates whether responses are correct or incorrect and gives feedback on incorrect answers. Students preferred this new alternative by a ratio of $61 \%$ to $39 \%$. Some individuals made favorable comments about effectively having an personal tutor, whereas others said that too much help was being provided.

Table 3. Relative Frequencies

\begin{tabular}{|l|c|c|c|c|c|c|c|c|}
\hline Method & a & b & c & d & e & f & g & h \\
\hline Frequency & $0 \%$ & $3 \%$ & $6 \%$ & $10 \%$ & $13 \%$ & $52 \%$ & $10 \%$ & $6 \%$ \\
\hline
\end{tabular}

\section{Alternative Paradigm}

The survey suggests that students should accept an approach to engineering homework similar to method $f$ or the alternative examined in question 3. The method examined in this paper is crafted with that in mind as well as addressing concerns of professors; it

- provides problems with the same structure, but different numbers;

- confirms when a student has entered a correct answer rather than giving the answer beforehand as a target;

- can provide solutions to similar problems (with different numbers) before the due date;

- can give the solutions to each student's problems after the due date; and 
- automates grading and provides other teacher-oriented aids.

This approach is part of a continuing evolution in using CAI for homework purposes. There are numerous examples, of which two are representative. Sullivan ${ }^{1}$ et all explored the use of web pages containing learning aids without randomization, and $\mathrm{Dahm}^{2}$ included random elements in a simulation based project for students. The current effort provides unique problems as the norm, thereby effectively offering a new paradigm for engineering homework.

In general, a major purpose of engineering homework is to insure that each student knows how to apply basic principles and integrate them in a manner that allows solving progressively realistic problems. Three example problems illustrate this.

1. Figure 3 applies a basic principle of engineering economics: If $D$ dollars are deposited at time $\mathrm{T}_{1}$ and earn interest at rate $i$, then what amount $\mathrm{W}$ can be withdrawn at $\mathrm{T}_{2}$ ?

2. Figure 4 shows a basic principle of statics: If $F_{1}, d_{1}$, and $d_{2}$ are known, then what must the value of $\mathrm{F}_{2}$ be to achieve equilibrium?

3. Figure 5 illustrates extending basic economic principles to solve more complex problems: Given an interest rate and deposits of amount $\mathrm{D}$ from year $\mathrm{T}_{1}$ through $\mathrm{T}_{2}$, then what equal amounts can be withdrawn from year $\mathrm{T}_{3}$ through $\mathrm{T}_{4}$ ?

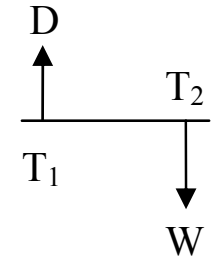

Figure 3. Basic Economics

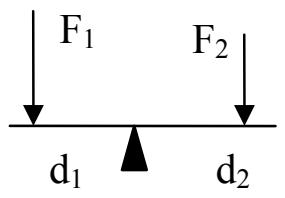

Figure 4. Basic Statics

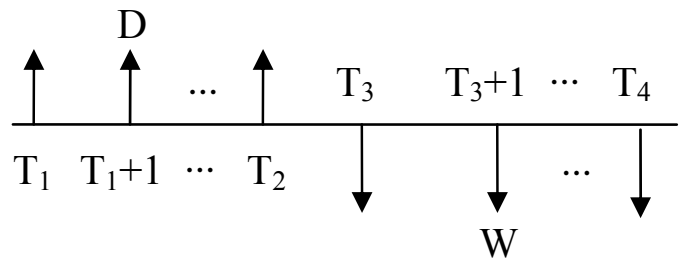

Figure 5. Extended Economics

In every case, it is the structure of these problems that is central to the learning experience, not the specific numeric values. The proposed paradigm consists of assigning problems with the same structure, but with unique inputs. This practice can lead to the following desirable consequences:

- Simple copying is not be feasible.

- An attempt to use homework files or postings of similarly structured problems requires understanding principles before applying them to a specific, unique problem.

- If there is collaboration, then the stronger student's desire to conserve time decreases the likelihood of the dependent student's receiving many complete solutions.

\section{Basic Implementation Issues}

Questions and answers on printed media do not work well for this new paradigm, but spreadsheets are well suited for it. They support text (including Greek symbols, subscripts, etc.) and graphics, and they can generate random numbers and perform extensive computations not well suited to a web page environment. For example, Figure 4 shows an Excel worksheet named Problem downloaded from a website. The question can be solved either on paper or on the computer, as in Figure 4. In cell C12 the student entered $=65^{\star} \mathrm{fp}(0.15,27-12)$ using the custom or user-written (by the professor) function $\mathrm{fp}$ in Table 4 to evaluate the engineering economics fac- 
tor $\mathrm{F} \mid \mathrm{P}$. (Code is provided for the those so inclined, but reading it is not necessary to understand the system.) It is best to require using numbers in computer solutions, such as 65 or 0.15 , rather than cell references to make copying difficult. Whether a paper or computer solution is required, the student must put the answer in a cell designated by the professor if automated grading is desired..

The original worksheet created by the professor includes the formulas shown in Figure 7, but the student never sees those formulas. When the student opens the workbook, Excel generates random

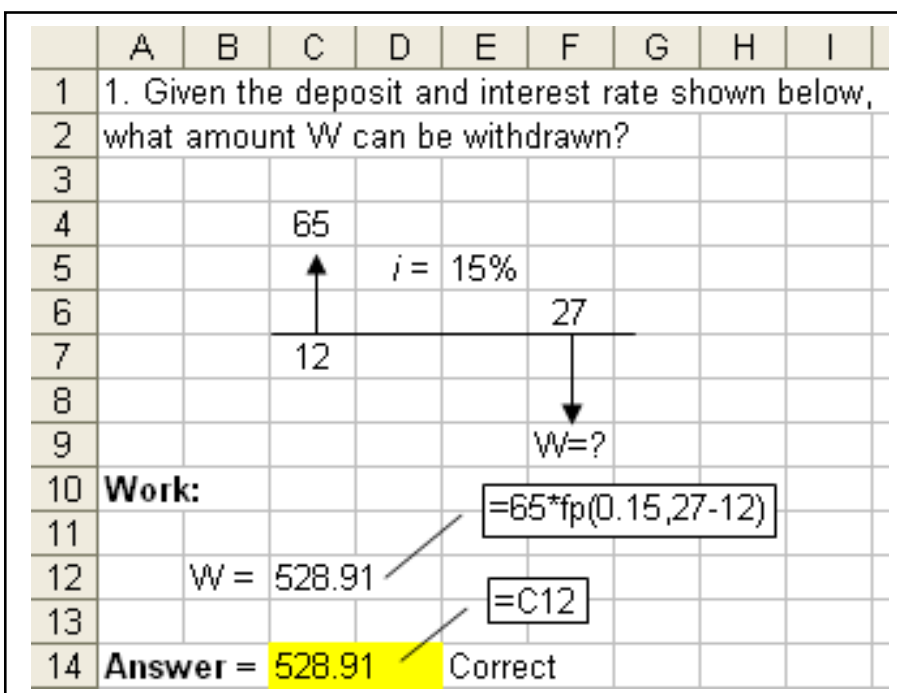

Figure 6. Spreadsheet Problem Seen by Student numbers requested by the custom function hg (in Table 5) that are based on the current time and date. Then the lengthy macro in Appendix A requests the student's identification, finds cells with formulas generating random numbers, replaces each of those formulas with its current value, and protects the values so they cannot be changed. The student is granted access to the workbook only after this process is completed.

The function $\mathrm{hg}$ is a random number generator that returns a value between a low and high parameter, and an optional third parameter can allow only discrete values. For example, the function's value in cell C4 can vary between 50 and 100 in steps of 5 , whereas the value in $\mathrm{E} 5$ can be between 0.08 and 0.20 in

Table 4. Custom Function fp

Function $\mathrm{fp}$ (Rate As Double, N As Integer)

'Evaluate the $\mathrm{F} \mid \mathrm{P}$ factor for Rate and $\mathrm{N}$ periods

If $\mathrm{N}<=0$ Then

MsgBox "Last parameter must be positive."

Exit Function

End If

$\mathrm{fp}=(1+\text { Rate })^{\wedge} \mathrm{N}$

End Function steps of 0.01 .

The custom function isclose in cell E14 compares a student's solution in cell $\mathrm{C} 14$ with the professor's solution in cell G10 of sheet Solutions. It allows for round-off error by displaying Correct if the student's answer is within either an absolute error of 0.5 or an absolute percent error of 0.01 ; otherwise, it returns Incorrect. Formatting the protection feature of cell E14 to Hidden and then protecting the sheet prevents the student from

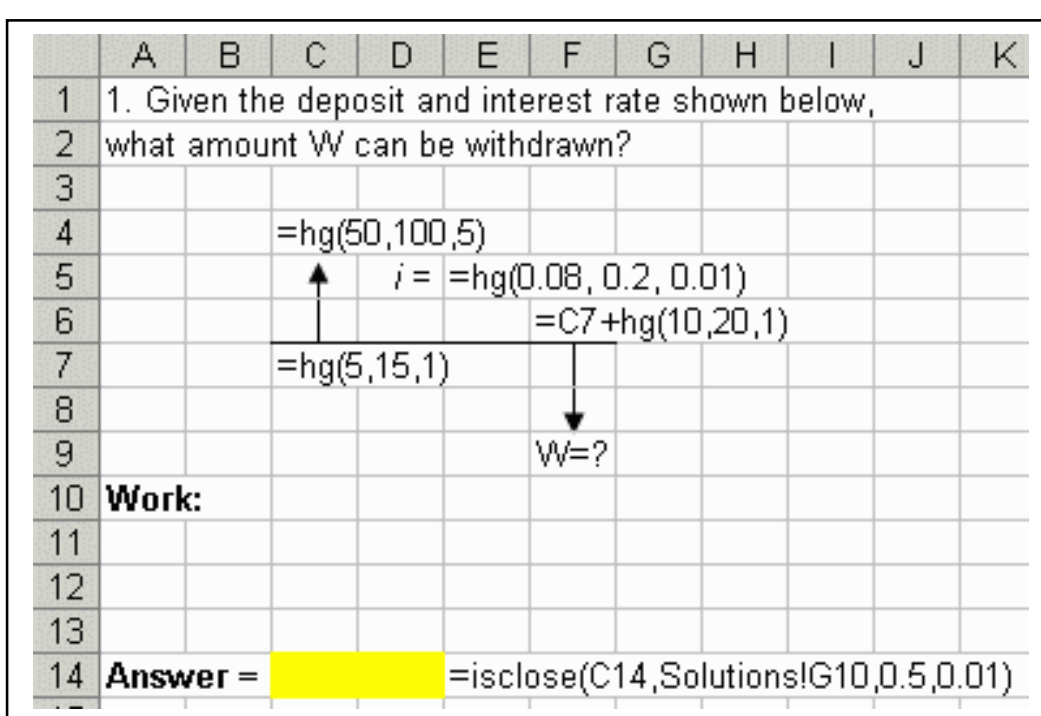

Figure 7. Worksheet Problem with Professor's Formulas 
Table 6. Random Number Generator

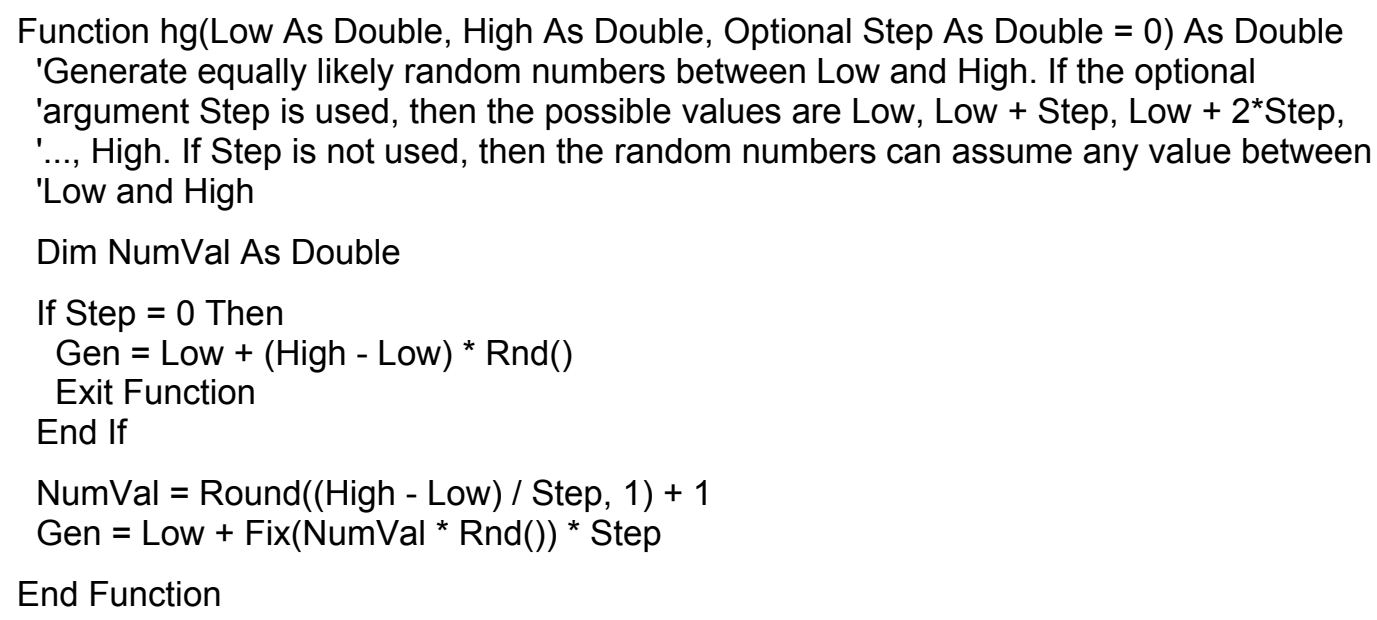

End Function

seeing the formula in cell E14, or any others that the professors does not want to show.

The foregoing techniques can be effected using Excel or the free OpenOffice ${ }^{4}$ system, and they are not limited to a specific discipline. For example, Appendix B shows how to implement the statics problem shown in Figure 4. These procedures provide each student with unique problems that cannot be changed, as well as feedback on whether the problem has been worked correctly. If a professor chooses to post a solution to a problem with the same structure but different numbers, students still must understand the solution logic to obtain their own solutions.

\section{Table 7. IsClose Function}

Function IsClose(Response As Range, Target As Double, AE As Double, APE As Double) As String

'Returns Response if Response is close enough to Target; else returns Target.

If Response.Value = "' Then

IsClose $=" "$

Exit Function

End If

If $($ Abs(Response - Target $)<=\mathrm{AE})$ Then

IsClose $=$ "Correct"

Exit Function

End If

On Error GoTo IsCloseFail

If (Abs((Response - Target) / Target $)<=$ APE) Then

IsClose $=$ "Correct"

Exit Function

End If

IsCloseFail:

IsClose = "Incorrect"

End Function 


\section{Enhancements for Professors}

The routines given in the preceding tables and Appendix A allow implementation of the proposed paradigm, but how likely is that? Two limiting factors are the time required to develop comprehensive problem sets and a lack of incentives for excellence in teaching. This section examines enhancements under development as part of the new $\mathrm{HmWk}$ system $^{5}$ and as extensions to $\mathrm{Coach}^{3}$ that reduce implementation time, and the next section addresses the issue of incentives.

Developing good homework problems requires creativity, and preparing questions and solutions for student use consumes time. Formatting text so that students see familiar notation can be tedious when using a word processor and daunting in a spreadsheet environment with randomly changing values, such as the subscript in cell D2 of Figure 8. Also providing even the simple graphics shown in Figure 3 through Figure 5 can take longer than anticipated. Fortunately, macro capabilities of spreadsheets can facilitate those tasks, and the spreadsheet environment can be exploited to recapture some of a professor's development time by automating grading and helping students having difficulty. Each of these capabilities is examined below.

\begin{tabular}{|c|c|c|c|c|c|}
\hline & A & B & C & D & $E$ \\
\hline 1 & 10 & 15 & & & \\
\hline 2 & $\mathrm{~B} 2 \sim \mathrm{D} 2 \sim=$ & E_L10 & & $E_{10}=$ & 20.00 \\
\hline 3 & \multicolumn{5}{|c|}{ 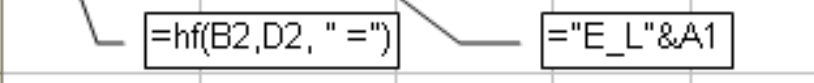 } \\
\hline \multicolumn{6}{|c|}{ Figure 8. hf Formatting Function } \\
\hline
\end{tabular}

\section{Notation and Formatting Text}

Word processors and spreadsheets insert hidden codes into text to display Greek symbols, subscripts, superscripts, and other formatting. Users select the text to be formatted and then choose a succession of menu items. For example, the entry E10 (a cash flow equivalent at time 10) is converted to $E_{10}$ in Excel by selecting the 10 and then successively clicking Format $\mid$ Cells $\mid$ Font $\mid$ Subscript. However, Excel requires E10 to be a string constant such as something typed into a cell rather than evaluated as a result of a formula such as = "E" \& A1, where A1 contains a random number that currently evaluates to 10 . Figure 8 shows how the custom function hf of HmWk handles this challenge.

The entry = "E_L" \& A1 in cell $B 2$ displays as $E \_L 10$, where the _L is a formatting code for subscripts. Then entering $=\mathrm{hf}(\mathrm{B} 2, \mathrm{D} 2$, " $=$ ") in A2 causes the contents of B2 to be placed into cell D2 as a string constant, formatted, and followed by an equal sign. (Usually a custom function cannot change any cell other than the one in which it appears, but this is circumvented by triggering an event, and using the event routine to change other cells.) The display in A2 denotes this process. Later, columns A and B can be hidden, so that only the desired formatted text is shown. There are formatting codes for every common need, and the time required to obtain dynamic, formatted results is little more than that for formatting static, non-random strings.

Figure 9 illustrates extending the basic concepts of formatting. The first two rows are as before, and cells $A 3: B 3$ cause $E_{15}=$ to display $n$ cell D3 as was done on row 2. Cell E3 contains the formula $=h v(E 2, B 2)^{\star} f p(10 \%, h v(B 1)-h v(A 1))$. The hv function in cell E3 returns the value of the first parameter, and the optional second parameter identifies the cell containing the first parameter's symbol. If hv has only one parameter, then it contains both the value and the symbol. As before, 


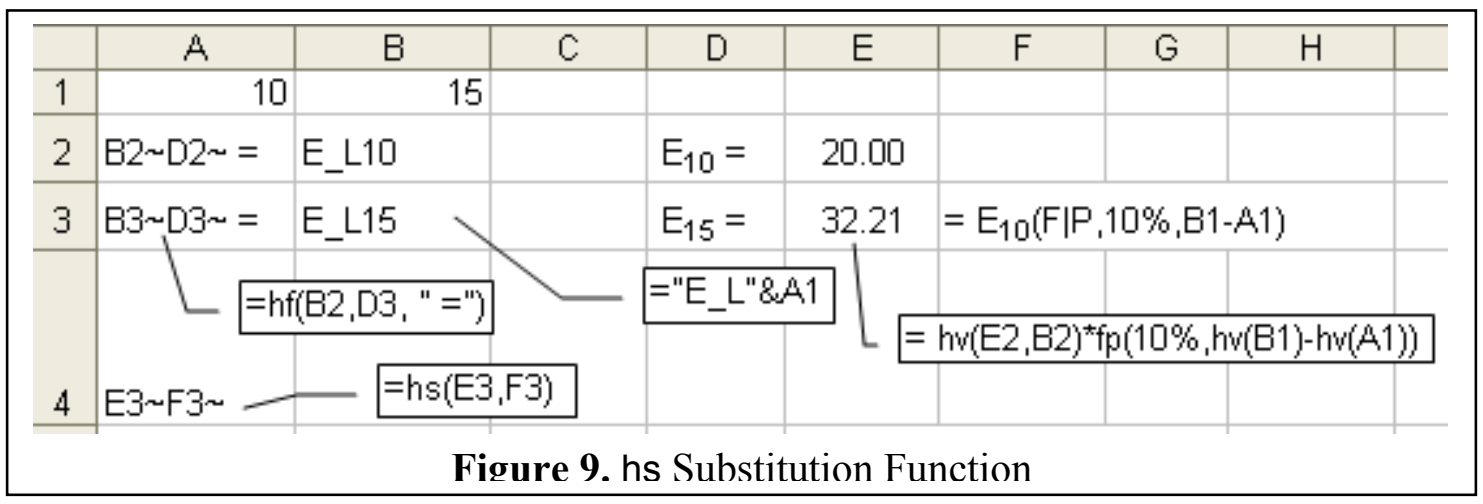

the fp function evaluates the F|P factor. The entry $=h s(E 3, F 3)$ in cell A4 uses the code shown in Table 8 to perform the following substitutions on the formula in E3 before putting the formatted result into cell F3:

- Any hv function is replaced by the instructions for its symbol, so $\mathrm{hv}(\mathrm{E} 2, \mathrm{~B} 2)$ is becomes the contents of B2, and B1 and A1 are replaced by their values.

- Entries on a substitution list stored in the workbook are replaced. Since $\mathrm{fp}$ ( is on the engineering economics substitution list shown in Figure 10, (F|P, is substituted for it. The substituted string can contain format codes, including those that provide Greek fonts.

\begin{tabular}{|c|c|}
\hline \multicolumn{2}{|c|}{ Substitution List } \\
\hline From & To \\
\hline $\mathrm{pf}($ & $(\mathrm{P} \mid \mathrm{F}$, \\
\hline $\mathrm{fp}($ & $(\mathrm{FIP}$, \\
\hline & \\
Figure 10. \\
Substitution List \\
\hline
\end{tabular}

The final product would show the student only the entries in D2:F4 by hiding columns A and B.

\section{Graphics}

Substitutions and graphics tend to be discipline specific. Cash flow diagrams are a primary need

Table 8. Custom Function hs

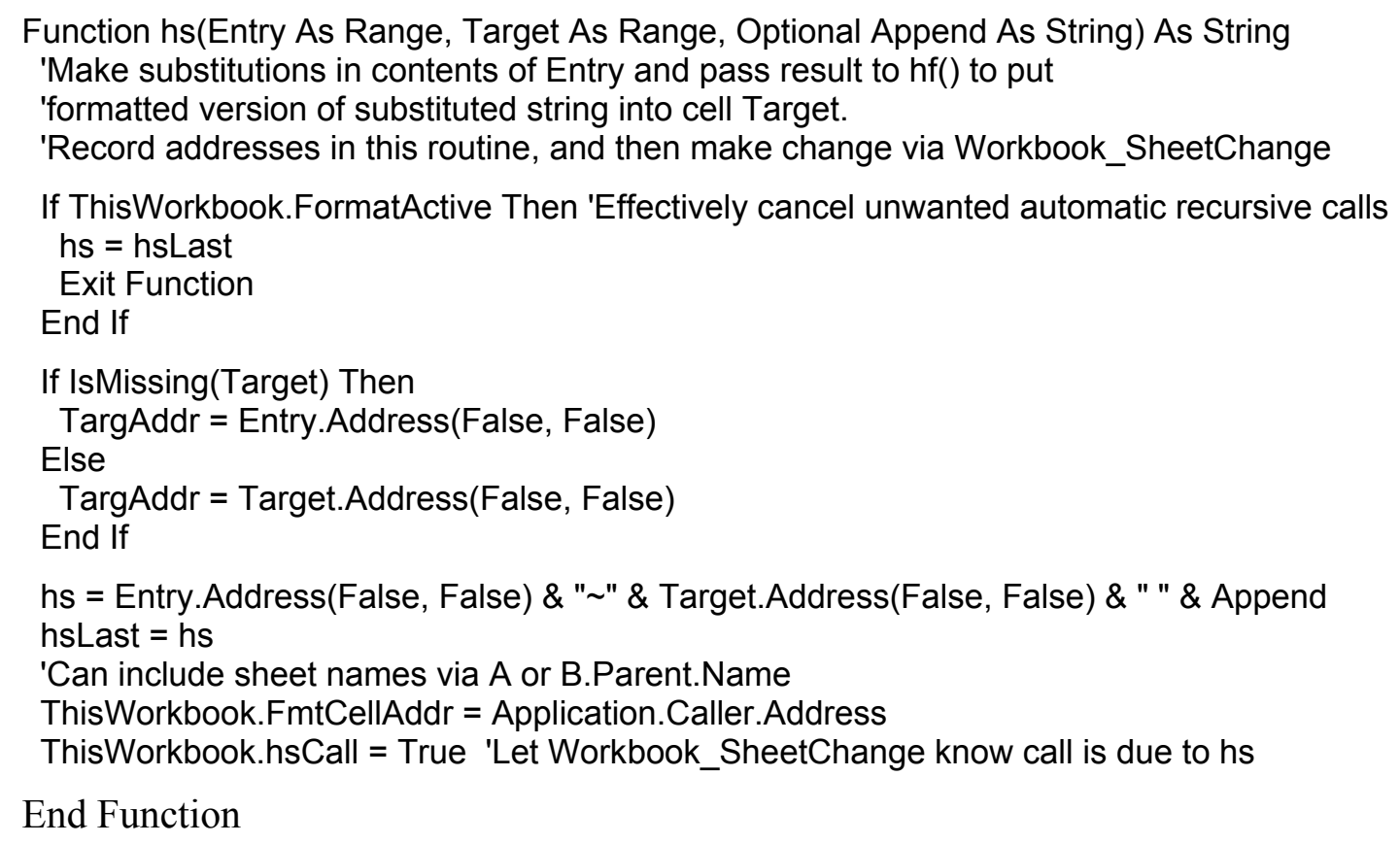




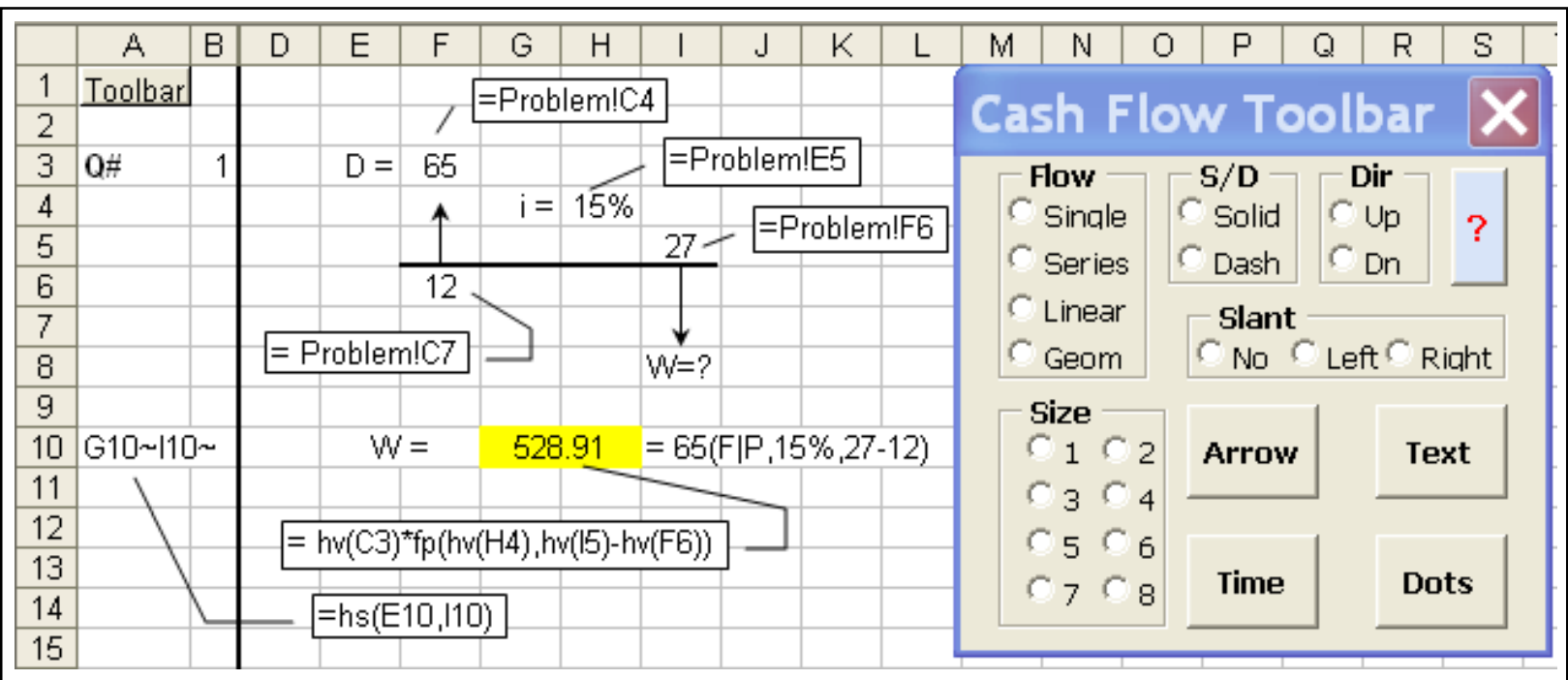

Figure 11. Graphics Toolbar and Professor's Entries on Sheet Solution

in engineering economics, and they consist of arrows, lines, and text. Once the graphics needs of a discipline are determined, then they can be addressed via custom toolbars or user forms. Figure 11 shows a graphics form for engineering economics that can be activated by clicking the command button in cell A1. Drawing one or more arrows is done by selecting the cells where the foot of each arrow will be drawn, and then clicking arrow properties:

- the type of flow (single flows, uniform series, linear and geometric trends),

- a size number (that will be multiplied by $1 / 3$ of the foot cell's height),

- $\quad$ and solid/dash, up/down, and slanting options.

Clicking the Draw button draws the arrows. Multiple flows such as series generally have two initial arrows and then a terminating arrow, and clicking the Dots button provides an ellipsis ( $\cdots$ ) to indicate the presence of a pattern. Similarly, time lines are created by selecting cells and clicking the Time button to put a line at the bottom of those cells. A figure's text can be typed into any cell and formatted as desired. For example, the hs entry in A10 processes the formula in G10 and places the result in I10.

\section{Automated Solutions and Grading}

The spreadsheet environment allows solutions, complete with graphics, to be provided for each student's unique problems. For example, Figure 11 is a screen capture of sheet Solutions, and it provides the solution of the question on sheet Problem shown in Figure 6. The problem data in cells F3, H4, I5, and F6 on sheet Solution all link back to sheet Problem by including the name Problem followed by an exclamation point. This allows the IsClose function in cell E14 of sheet Problem to compare the student's and professor's answers. Worksheet Grades in Figure 12 uses this capability to automate the grading process.

Cells A1:A3 contain the student's identity declared when the workbook opened, and cell A4 is the date and time that it was opened. (If two students have the same random numbers but differ- 


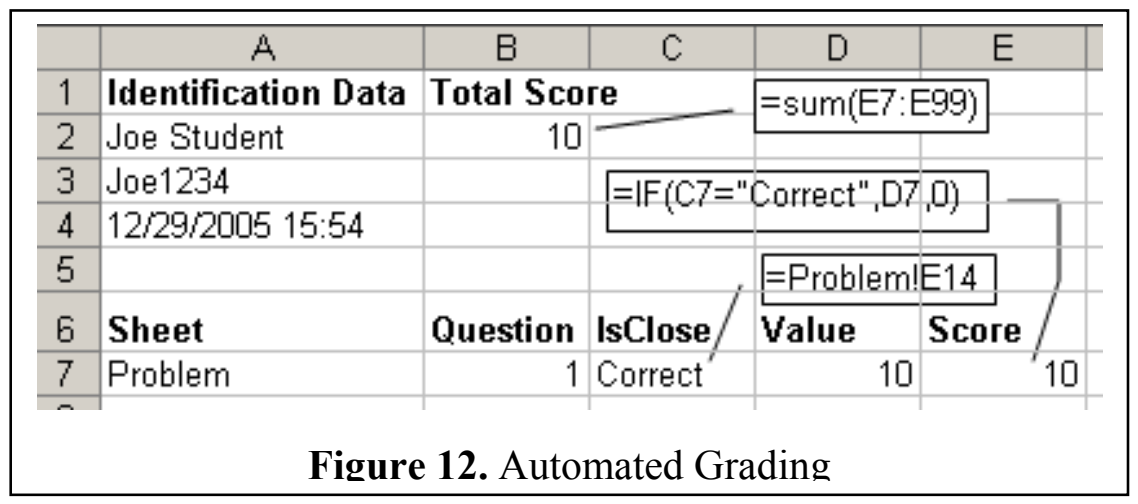

ent date/time entries, then there is clear evidence of copying from one workbook to another.) Column C contains links to IsClose functions, and column D stores the points assigned to each question by the professor. Column E uses IF functions to assign the student's score on each question, and cell B2 totals those scores. If students have access to solutions of similar problems and feedback regarding the correctness of their solutions, then it there should be no problems with assigning a homework grade based solely on the correctness of the answer.

\section{$\underline{\text { Help }}$}

At a minimum, professors still can provide help for students using traditional office hours. Similarly, generic solutions can be posted either physically or on a website. This paradigm offers a new wrinkle with regard to posting, because now students cannot simply copy the posts. Unique problems force understanding of a problem's logic to obtain a correct answer. If a professor has prepared complete solutions for each unique problem as shown in Figure 11, then the solution sheet can be hidden, password protected, and included in the download. Posting the password after the homework is submitted allows each student to review his or her own unique problems. In short, the entire focus of the learning process shifts from numbers to logic.

Interactive learning systems such as $\mathrm{Coach}^{3}$ can provide more help in the manner described in question 3 of the survey, at the cost of more time on the part of the professor. This Excel-based system displays a problem such as the one shown in Figure 6, and then requests the student to enter an answer (via typing, drop-downs, or clicking). Correct answers receive some form of congratulations and awarding of points. Incorrect answers initiate a series of steps designed by the professor that consist of prompts, responses, feedback appropriate to each response, and control over which prompt comes next as a function of the response. Upon completion of those steps for the current problem, Coach can change the random numbers and once again repeat the process, assigning points only if the student completes the question without aid. Coach minimizes the implementation effort required for this tutoring, but it is more than the protocol discussed above, so it probably would be a better focus with Coach on interactive examples than to try and use it for every homework problem.

\section{Conclusion}

It is a fascinating irony that engineers are change agents who frequently resist change. Engineering homework is an absolutely crucial part of the learning process, but it really has not advanced 
during the last century, other than abandoning slide rules for more efficient computational aids. This paper proposes a new paradigm to benefit from the information age that engineers helped to create: Each student receives problems having the same logical structure but with unique numbers. This forces a focus on analysis and logic, and it encourages each student to reach his or her own potential by squarely addressing long-standing problems of copying or working together too closely.

Change is rarely easy, but this is a concept whose time has come. Students clearly recognize its benefits, and this paper establishes its technological feasibility. Logistical problems of preparing unique questions and solutions have been solved, and enhancements for professors have been developed including formatting and notation, graphics, and automated grading and help for students. It is something that engineering education needs and should have, but it takes time, and spending too much time on teaching can be harmful to a professor's career unless there is administrative support.

One simple, very real reason why some managers of engineering education emphasize research at the expense of teaching excellence is that research is easier to measure. Department chairs conducting performance reviews quickly learn that evaluating the quality of teaching can lead to very long and painful days, and deans and higher administrators have progressively fewer means to objectively evaluate teaching. It is easier to count articles and much-needed dollars, and measurable achievement helps a manager to advance, just as it does for a professor.

This new paradigm does not guarantee that a professor will do a good job in the classroom, but it does provide teaching metrics every bit as valid as counts of articles or dollars. For example, how many problems have been made available for students? This, in turn, suggests other measures such as web page creation and the development of courseware. Moreover, the conceptual design such a CAI system is, in itself, an exciting scholarly activity; and students can be involved in that process and its implementation. There is no magic that will make either teaching or its evaluation easy, but this approach to a central element of engineering education offers an opportunity to provide a balance between teaching and research, as well as its pedagogical advantages.

A possible outcome of this paradigm is the creation of open-source websites for engineering professors that provide individualized problems and solutions in every discipline. The ASEE provides an excellent organizational structure for this endeavor. Divisional webmasters can attend to the needs of each discipline, and coordination among division chairs and professional interest councils can help develop guidelines and encourage software development that will enhance engineering education for both professors and students.

\section{Bibliography}

1. Terpenny, Janis P., William G. Sullivan, Harpreet Singh, and Kimberly Sward, "Utilizing the Internet to Improve Student Learning in a First Course in Engineering Economy with Real-World Unsolved Problems in Collaboration with Industry," Proceedings of the 2002 American Society for Engineering Education Annual Conference, http://www.asee.org/ conferences.

2. K. D. Dahm, "Interactive Simulation for Teaching Engineering Economics," Journal of SMET Education, March 2003.

3. Ristroph, John H., "Coach: A New System for Interactive Learning," presented and published as Proceedings of the ASEE National Conference, Portland, June, 2005. 
4. OpenOffice Software, http://www.openoffice.org/, December 2005.

5. Ristroph, John H., "HmWk: A System for Preparing Individualized Engineering Homework Problems and Solutions with Automated Grading," working paper.

\section{Appendix A: Initial Macro}

Private Sub Workbook_Open()

'Execute when workbook is opened to configure it for each student's use

Dim ChangeCell As Range, ShIndex As Integer, FirstTime As Boolean

Dim InputStr As String

Const Password As String = "myword" 'Change this as desired

'See if workbook being opened for first time

If Sheets(1).Range("D1") = "' Then

InputStr = InputBox("Please enter your full name:")

Sheets(1).Range("D1") = InputStr

FirstTime $=$ True

End If

'Change sheet for student's use

If FirstTime Then

ShIndex = ActiveSheet.Index 'Store index of active sheet to return to it later

'For every sheet in the workbook

For Each Sh In Sheets

Sh.Activate

Cells. Locked $=$ False 'Initially unprotect all cells

If Sh.Index = 1 Then Range("D1").Locked = True 'Lock cell with student's name

Range("a1").Select

'Find each cell w/ "=gen(", convert it to values, and then lock it

Do

Set ChangeCell = Cells.Find(What:="gen(", After:=ActiveCell, LookIn:=xIFormulas, LookAt:=xIPart, SearchOrder:=xIByRows, SearchDirection:=xINext, MatchCase:= False, SearchFormat:=False)

If ChangeCell Is Nothing Then Exit Do

ChangeCell.Select

Selection.Formula $=$ ChangeCell. Value

Selection.Locked $=$ True 'Lock cells that contain data inputs

Loop

'Protect locked cells

ActiveSheet.Protect Password:="myword", UserInterfaceOnly:=True

Next 'Sh in Sheets

Sheets(ShIndex).Activate 'Return to sheet initially seen by student

Range("a1").Activate

End If 'FirstTime

End Sub 


\section{Appendix B: Unique Statics Problem for Each Student}

\begin{tabular}{|c|c|c|c|c|c|c|c|c|c|c|c|}
\hline & A & $\mathrm{B}$ & $\mathrm{C}$ & D & $\mathrm{E}$ & $\mathrm{F}$ & G & $\mathrm{H}$ & 1 & J & K \\
\hline 1 & \multicolumn{11}{|c|}{ 1. Given the forces and distances shown below, what force } \\
\hline 2 & \multicolumn{7}{|c|}{ on the right balances the seesaw? } & & & & \\
\hline 3 & & & & & & & & & & & \\
\hline 4 & & & 72 & & & & & & & & \\
\hline 5 & & & 1 & & & & & & & & $x=?$ \\
\hline 6 & & & & & & & & & & & 1 \\
\hline 7 & & & 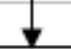 & & & & & & & & $\frac{1}{y}$ \\
\hline 8 & & & & 4.6 & & & $\boldsymbol{\Lambda}$ & & 5.6 & & \\
\hline 9 & & & & & & & & & & & \\
\hline 10 & & Wor & & & & & & & & & \\
\hline 11 & & & & & & & & & & & \\
\hline 12 & & & & & & & & & & & \\
\hline 13 & & Ans & $\mathrm{er}=$ & & & & & & & & \\
\hline & & & & $i$ & & & & & Sth & & \\
\hline
\end{tabular}

\begin{tabular}{|c|c|c|c|c|c|c|c|c|c|c|c|c|c|}
\hline & A & $\mathrm{B}$ & $\mathrm{C}$ & $\mathrm{D}$ & $E$ & $\mathrm{~F}$ & & $\mathrm{H}$ & 1 & $\mathrm{~J}$ & K & $\mathrm{L}$ & M \\
\hline 1 & \multicolumn{13}{|c|}{ 1. Given the forces and distances shown below, what force } \\
\hline 2 & \multicolumn{7}{|c|}{ on the right balances the seesaw? } & & & & & & \\
\hline 3 & & & & & & & & & & & & & \\
\hline 4 & & & \multicolumn{3}{|c|}{$=h g(60,100,1)$} & & & & & & & & \\
\hline 5 & & & 5 & & & & & & & & $x=?$ & & \\
\hline 6 & & & & & & & & & & & 1 & & \\
\hline 7 & & & 1 & & & & & & & & 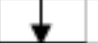 & & \\
\hline 8 & & & & \multicolumn{3}{|c|}{$=h \mathrm{~g}(3,5,0.1)$} & 7 & & \multicolumn{3}{|c|}{$=\mathrm{D} 8+h \mathrm{~g}(1,3,0.1)$} & & \\
\hline 9 & & & & & & & & & & & & & \\
\hline 10 & & \multicolumn{2}{|c|}{ Work: } & & & & & & & & & & \\
\hline 11 & & & & & & & & & & & & & \\
\hline 12 & & & & & & & & & & & & & \\
\hline 13 & & \multicolumn{2}{|c|}{ Answer $=$} & & & \multicolumn{8}{|c|}{$=$ isclose $(\mathrm{D} 13$, Statics Solutions!G10,0.5,0.01) } \\
\hline
\end{tabular}

Figure 14. Statics Spreadsheet Created by Professor

$$
\begin{aligned}
& \mathbb{D} \\
& \stackrel{0}{D} \\
& \vec{\Xi} \\
& \dot{\vec{J}} \\
& \dot{\vec{\sigma}}
\end{aligned}
$$

